\title{
Assessment of intensity modulated radiation therapy in left breast cancer including regional nodes without the internal mammary node: secondary cancer risks on thyroid and stomach
}

\author{
H. Jang',2", J.G. Baek', W. Kim¹, J.H. Sohn² \\ ${ }^{1}$ Department of Radiation Oncology, Dongguk University Gyeongju Hospital, Gyeongju, South Korea \\ ${ }^{2}$ Dongguk University College of Medicine, Gyeongju, South Korea
}

\section{- Original article}

*Corresponding author:
Hyunsoo Jang, Ph.D.,
E-mail:
$\quad$ opencagejhs@gmail.com
Revised: October 2020
Accepted: November 2020
Int. J. Radiat. Res., October 2021;
19(4): 921-928
DOI: 10.29242/ijrr.19.4.921

\begin{abstract}
Background: There is no clear guideline regarding the optimum intensity modulated radiation therapy (IMRT) technique for patients with breast cancer (BC) requiring radiotherapy (RT) treatment of the regional node area but not of the internal mammary node (IMN). We evaluated the IMRT technique with a focus on secondary cancers of stomach and thyroid. Materials and Methods: Eight patients with left BC treated with RT after breast conserving surgery at a single institution in 2017 were enrolled. Three-dimensional conformal radiation therapy (3D-CRT) consisting of two opposed half tangential breast fields and IMRT plans was performed. Normal organ dosimetric parameters were compared. Excess absolute risks, excess relative risks, and lifetime attributable risks (LAR) were calculated. Results: Stomach V30 values were 10.27 and 1.31 for tangential 3D-CRT and IMRT, respectively, and corresponding V40 values were 7.46 and 0.2 , whereas V5 values were 21.15 and 49.62, respectively. Thyroid values were similar; V30 26.53 and 7.93 , V40 22.37 and 2.63, and V5 40.93 and 88.86, respectively. LAR values of stomach were 1.76 (per 100 persons) and 2.31 and for thyroid were 5.3 and 9.5, respectively. LAR values of contralateral breasts were 0.35 and 0.99 , of ipsilateral lungs were 1.68 and 2.39, and of contralateral lungs were 0.58 and 1.73. All values weresignificantly different $(p<0.05)$. Conclusion: $L A R$ values of stomach and thyroid were higher for IMRT than 3D-CRT in left BC patients requiring regional node treatment without including IMN. Consensus on the priority among disease control rate, secondary cancer risk, and toxicity is required.
\end{abstract}

Keywords: Radiotherapy, Breast cancer, Second cancer, Intensity modulated radiation therapy.

\section{INTRODUCTION}

Breast cancer (BC) is a highly prevalent female cancer and is treated by combined surgery, radiotherapy (RT), chemotherapy, and hormone therapy according to tumor location, size, and disease stage (1). Today, breast conserving surgery and RT is recognized as a standard treatment for almost all early and some locally advanced BCs (2). Various RT techniques are used which differ in terms of the method used to deliver radiation. The tangential field-infield (FIF) technique is traditionally used, though recently, the intensity modulated radiation therapy (IMRT) technique is being actively investigated in $\mathrm{BC}$ (3).

The choice of RT technique for $\mathrm{BC}$, especially for left $\mathrm{BC}$, is somewhat controversial. In early stage $\mathrm{BC}$, RT based on conventional tangential techniques is commonly used. In the locally advanced stage, which includes the internal mammary node (IMN), IMRT is used to reduce 
exposure of lungs and heart to high doses $(4,5)$. However, in patients that require regional node treatment but not IMN treatment, there is no clear guideline regarding the IMRT technique of choice, which is invariably determined by clinician judgment and experience. Therefore, to implement IMRT in these patients, the merits and demerits of IMRT should be clarified. In practice, RT technique tends to be selected with a focus on lung and heart toxicity, and it effects on adjacent organs such as stomach and thyroid are often overlooked. If IMN is included in the scope of treatment, there is ample evidence that the IMRT technique can reduce lung and heart toxicity (4), but in other cases the benefits of IMRT should be accurately assessed.

In situations where the risk of acute or chronic RT toxicity is not high, the probability of RT-induced secondary cancer (SC) may be important when selecting RT technique. Adjacent organs, such as stomach and thyroid, are often included in planning target volumes (PTVs) and are exposed to significant amounts of radiation, which can greatly increase the probability of SC $(6,7)$, although it should be added that in practice, the SC risk usually not considered when choosing RT techniques. Previous studies on SC have been largely phantom studies on scattered doses beyond the irradiation field (8-10), and relatively few studies have addressed exposure doses near irradiation fields. One reason for this lack of study is that it is difficult to specify the exposure dose due to the presence of a large dose gradient near the irradiation field. However, this problem could be overcome by calculating organ equivalent doses (OEDs) (11).

Although IMRT technique is useful and actively attempted in $\mathrm{BC}$, the SC risk is often ignored. And in $\mathrm{BC}$ whose IMN is negative, the IMRT technique tends to be excessively applied, even though it can perform well enough with the conventional tangential technique. The purpose of present study was to analyze the gain and loss of the IMRT technique in patients with left BC requiring $\mathrm{RT}$ treatment of the regional node area but not of the IMN. In particular, we focused on the risk of SC in adjacent organs, evaluated risks of SC in stomach and thyroid, and attempted to quantify these risks.

\section{MATERIALS AND METHODS}

\section{Patients and treatment planning}

Eight patients with left breast cancer treated with RT after breast conserving surgery at a single institution in 2017 were enrolled. The nodal stage of all eight patients was $\mathrm{N} 1$, and thus, the axillary lymph node and supraclavicular lymph node were included in the RT field, but not the IMN. Patient information is provided in table 1.

All patients underwent planning computed tomography (CT) using a Toshiba Asteion helical CT scanner (Toshiba Medical Systems, Tokyo, Japan) for RT planning and clinical target volumes (CTV) were contoured according to the Radiation Therapy Oncology Group (RTOG) guideline, and the PTVs were $5 \mathrm{~mm}$ beyond CTVs; volumes that extended beyond the skin or included lung were excluded from PTVs.

Organs at risk (OAR) were defined as stomach, thyroid, contralateral breast, contraand ipsilateral lung, and entire heart. All plans used in the study were generated using the Varian Eclipse planning system using a $6 \mathrm{MV}$ photon beam and doses were prescribed to PTV according to the dose scheme of 50 Gy in 25 fractions. Three-dimensional conformal radiation therapy (3D-CRT) FIF consists of two opposed half tangential breast fields with 1-3 subfields on each side and a single anterior supraclavicular field. Beam angles, number of fields, and beam weightings were optimized for PTV coverage. For IMRT plans, the sliding window technique with 5 to 6 fields was adopted using the same PTVs used in 3D-CRT plans (figure 1).

Analytical anisotropic algorithm (ver. 8.9, Varian Medical Systems) and progressive resolution optimizer (ver. 8.6, Varian Medical Systems) were used to predict patient dose distributions in IMRT plans. Dose constraints for normal organs were based on RTOG 0623. This study was approved by our institutional review board (110757-201811-HR-03-02) and was conducted in accordance with the principles of 
the Declaration of Helsinki.

\section{Organ dose}

Organs near the irradiation field lie in large dose gradients, which makes it difficult to quantify the dose applied, and thus, an OED linear-exponential model was applied (11). Organ volumes exposed to each dose at 1 Gy intervals were obtained using the Eclipse program and applied to the equation (1). Using this formula, organ exposures were calculated as single values.

$$
\mathrm{OED}=\frac{1}{V} \sum_{i} D V H\left(D_{i}\right) \cdot D_{i} \cdot e^{-a D i}
$$

Where DVH(Di) is the volume receiving dose $\mathrm{Di}$ and $\mathrm{V}$ is total organ volume. The constant $\alpha$ is $0.044 \mathrm{~Gy}^{-1}$ and was estimated by fitting to the Japanese atomic bomb and Hodgkin cohorts. OEDs were defined as total doses, and thus, modified OED (MOED) values were calculated that considered biologically effective dose (BED) (2), to compensate for the biologic effects caused by dose fractionation ${ }^{(12) .}$

Biologically effective dose $=(n d)(1+d /(\alpha / \beta))(2)$

Where $\mathrm{n}$ and $\mathrm{d}$, are total fraction number and fraction dose, respectively; the $\alpha / \beta$ ratio was set at 3 Gy.

\section{Dosimetric parameters}

Normal organ dosimetric parameters for stomach, thyroid, lung, and contralateral breast were compared using RT planning techniques. The dose volume histogram (DVH) of all plans obtained, and dosimetric parameters, such as $\mathrm{V}_{\text {dose }}$ and mean dose, were calculated from DVHs. $V_{\text {dose }}$ was defined as the percentage volume that received at least the dose. In addition, monitor units (MUs) were calculated, and homogeneity indices (HIs) were evaluated (HI was defined as: $\left(D_{2}-D_{95}\right) / D_{50}$, where $D_{n}$ is the minimum dose in $n$ $\%$ of a PTV, as described in ICRU-83 (13)).

\section{Estimation of secondary cancer risk}

Excess absolute risk (EAR), excess relative risk (ERR), and lifetime attributable risk (LAR) were calculated using the Biological Effects of Ionizing Radiation (BEIR) VII model. ERR was defined as excess risk with respect to background risk, and EAR as the difference between total and background risk (14). The equation (3) for EAR and ERR is:

EAR and $\operatorname{ERR}(D, e, a)=\beta s \times D \times \exp (\gamma e)(a / 60) \eta$

Here, $\mathrm{D}$ is mOED, e is age at exposure, and a is attained age; $\beta s, \gamma$, and $\eta$ are model parameters. A latency period of 5 years was used for solid cancer, and attained age (a) was defined as the sum of $e$ and latency period. LAR is the probability that an exposed person will develop a malignancy during his/her lifetime. The equation (4) for LAR is:

$$
\begin{aligned}
& \left(\sum_{a}^{90} \operatorname{ERR}(D, e, a) \cdot \lambda^{C} \cdot \frac{S(a)}{S(a) d a}\right) \mathrm{w} \times \\
& \left(\sum_{a}^{90} \operatorname{EAR}(D, e, a) \cdot \frac{S(a)}{S(a) d a}\right) 1-\mathrm{w}
\end{aligned}
$$

where $\mathrm{w}$ is weight, $\lambda c$ is baseline cancer risk, and $\mathrm{S}(\mathrm{a}) / \mathrm{S}(\mathrm{e})$ is the probability of surviving at age $a$ if the patient survives to age e. Baseline cancer risks and life spans used were in accord with the Korea Central Cancer Registry 2015 and Statistics Korea 2017, respectively. For stomach cancer and lung cancer, $\mathrm{w}$ values were 0.7 and 0.3 , respectively. The ERR model was used for thyroid cancer and the EAR model for breast cancer $(14,15)$.

Table 1. Patient characteristics.

\begin{tabular}{|c|c|c|c|c|c|c|}
\hline No & Age & Stage & Operation & Radiotherapy & Pathology & Chemotherapy \\
\hline 1 & 46 & T2N1M0 & BCS, ALND & $50 \mathrm{~Gy} / 25 \mathrm{fx}$ & IDC & AC\#4, T\#4 \\
\hline 2 & 35 & T1N1M0 & BCS, SLNB & $50 \mathrm{~Gy} / 25 \mathrm{fx}$ & IDC & AC\#4, T\#4 \\
\hline 3 & 64 & T2N1M0 & BCS, SLNB & $50 \mathrm{~Gy} / 25 \mathrm{fx}$ & IDC & AC\#4, T\#4 \\
\hline 4 & 45 & T2N1M0 & BCS, SLNB & $50 \mathrm{~Gy} / 25 \mathrm{fx}$ & IDC & AC\#4, T\#4 \\
\hline 5 & 47 & T1N1M0 & BCS, SLNB & $50 \mathrm{~Gy} / 25 \mathrm{fx}$ & IDC & AC\#4, T\#4 \\
\hline 6 & 52 & T1N1M0 & BCS, ALND & $50 \mathrm{~Gy} / 25 \mathrm{fx}$ & IDC & AC\#4, T\#4 \\
\hline 7 & 42 & T2N1M0 & BCS, ALND & $50 \mathrm{~Gy} / 25 \mathrm{fx}$ & IDC & AC\#4, T\#4 \\
\hline 8 & 42 & \multicolumn{7}{|c|}{ T2N1M0 } & BCS, ALND & $50 \mathrm{~Gy} / 25 \mathrm{fx}$ & IDC & TC\#6 \\
\hline BCS, Breast Conserving Surgery; ALND, Axillary Lymph Node Dissection; SLNB, Sentinel Lymph Node Biopsy; IDC, \\
invasive ductal carcinoma; AC, doxorubicin and cyclophosphamide; T, taxol; TC, taxol and cyclophosphamide.
\end{tabular}




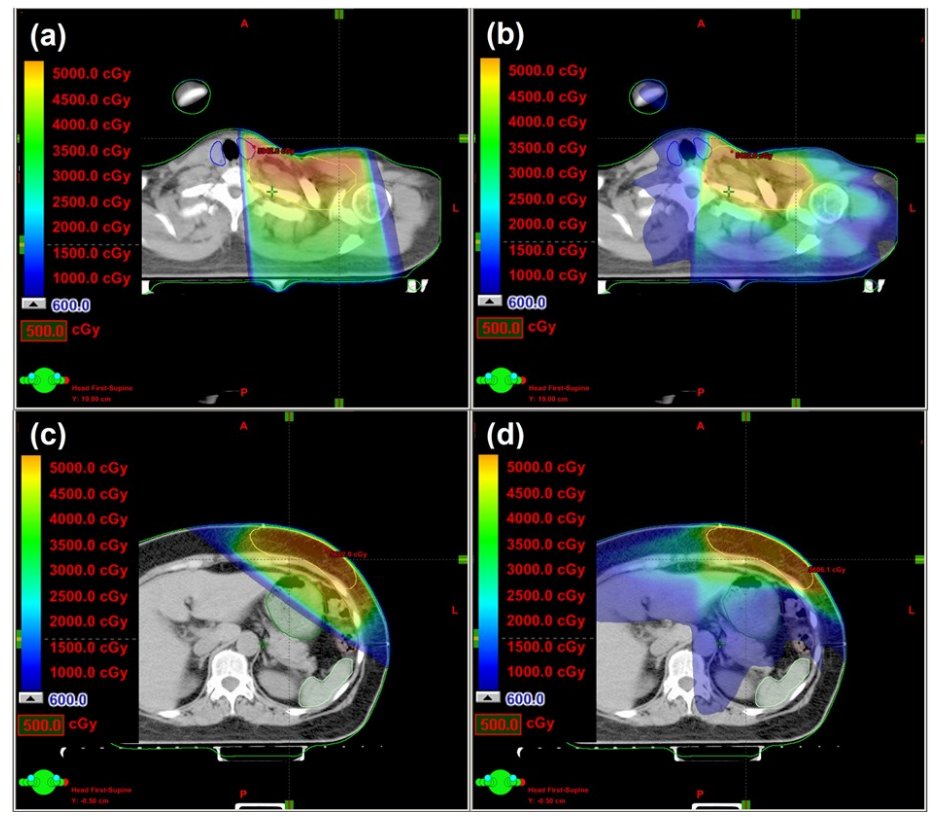

Figure 1. Comparison of irradiated isodose distributions in the transversal planes of supraclavicular node (SCN) and breast regions for the two treatment techniques. (a) SCN for 3D-CRT, (b) SCN for IMRT, (c) breast for 3D-CRT, (d) breast for IMRT.

\section{Statistically analysis}

Wilcoxon's signed rank test was used to compare dosimetric results, and SPSS version 20 (SPSS Inc., Chicago, IL) was used. Statistical significance was accepted for $\mathrm{p}$ values $<0.05$.

\section{RESULTS}

The mean organ volumes of stomach, thyroid, contralateral breast, contralateral lung, and ipsilateral lung were 256.71, 13.39, 347.57, 923.57, and $1142.34 \mathrm{cc}$, respectively. V30 values for stomach were 10.27 and 1.31 for tangential 3D-CRT and IMRT, respectively, and corresponding V40 values were 7.46 and 0.2 (p $<0.05)$, whereas V5 values were 21.15 and 49.62, respectively. Thyroid and stomach results were similar; V30 26.53 and 7.93, V40 22.37 and 2.63, and V5 40.93 and 88.86, respectively (tangential 3D-CRT vs. IMRT, $p<0.05$ ). In contralateral breast, high doses of V30 and V40 were all less than 1\%, but V5 values of tangential 3D-CRT and IMRT were significantly different at 1.38 and 42.06 , respectively. The V40 values of ipsilateral lung were 37.81 and 17.87, and V5 values were 59.88 and 93.71, respectively. The V30 and V40 of contralateral lung were not detected all for tangential 3D-CRT and IMRT, and V5 values were 0.04 and 34.49, respectively. Details are given in figure 2 and table 2.

mOEDs for stomach were significantly different at 1.94 and 2.54 Gy for tangential 3D-CRT and IMRT, respectively. mOEDs of thyroid were $1.85 \mathrm{~Gy}$ and $3.27 \mathrm{~Gy}$, of contralateral breast were $0.87 \mathrm{~Gy}$ and $2.51 \mathrm{~Gy}$, of ipsilateral lung were $2.34 \mathrm{~Gy}$ and $3.32 \mathrm{~Gy}$, and of contralateral lung were 0.81 Gy and 2.42 Gy, respectively. The OEDs and mOEDs for each organ are summarized in Table 3.

LAR values of organs were calculated using mOEDs. LARs of stomachs were 1.76 (per 100 persons) and 2.31 for tangential 3D-CRT and IMRT, respectively, of thyroid were 5.3 and 9.5, of contralateral breast were 0.35 and 0.99 , of ipsilateral lung were 1.68 and 2.39, and of contralateral lung were 0.58 and 1.73, respectively. Tangential 3D-CRT and IMRT LAR values were significantly different for each organ. ERR, EAR, and LAR values for each organ are summarized in table 3.

Mean MU values were 475.88 and 1369.75 for tangential 3D-CRT and IMRT, respectively, and mean $\mathrm{HI}$ values were 0.29 and 0.14 , and both $\mathrm{MU}$ and $\mathrm{HI}$ values were significantly different. MU and $\mathrm{HI}$ values for individual patients are provided in table 4 .

Int. J. Radiat. Res., Vol. 19 No. 4, October 2021 

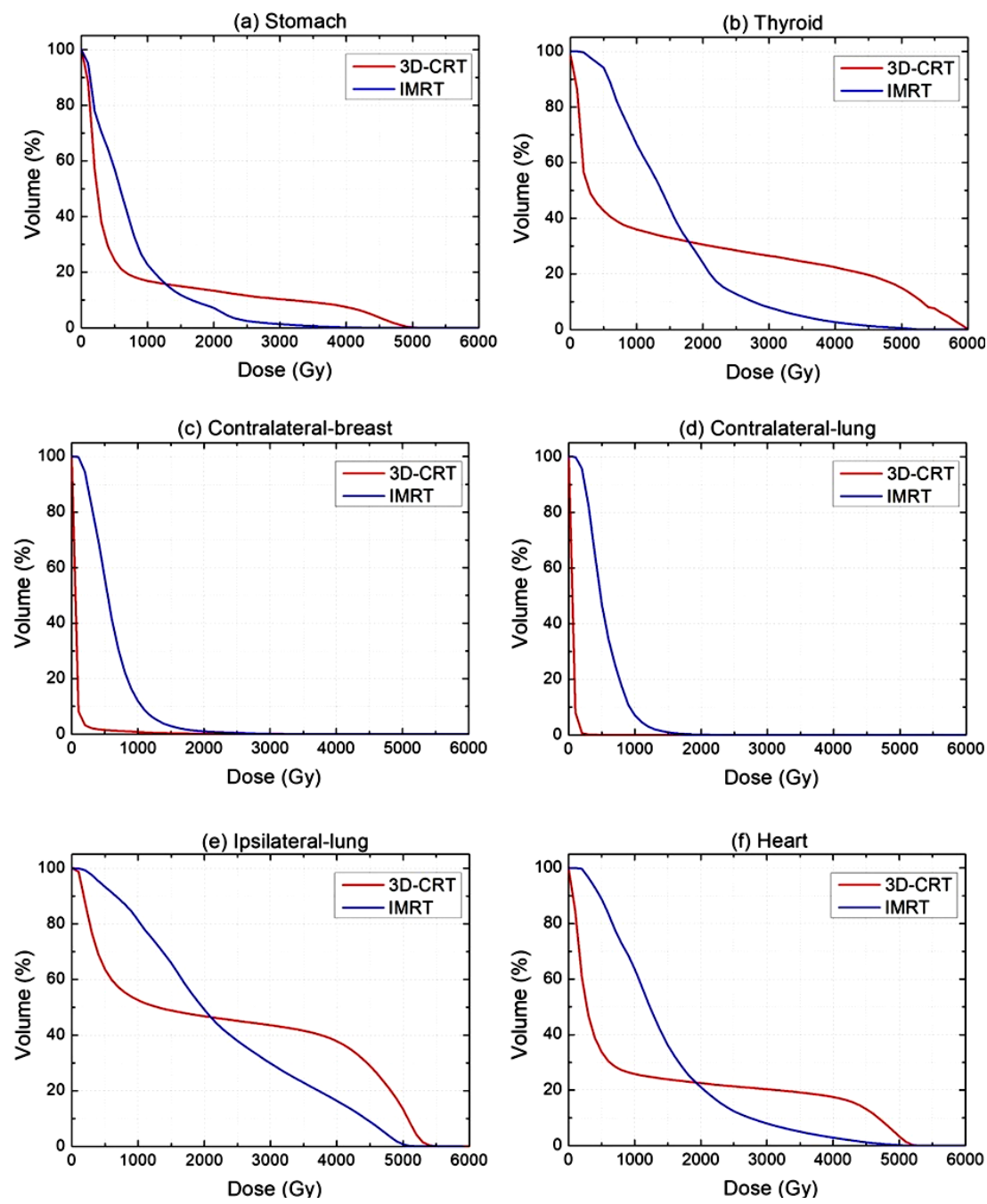

Figure 2. Mean dose-volume histogram comparison for 3D-CRT and IMRT plans. (a) Stomach, (b) thyroid, (c) contralateral-breast, (d) contralateral-lung, (e) ipsilateral-lung, (f) heart.

Table 2. Mean dosimetric parameter values

\begin{tabular}{|c|c|c|c|c|c|c|}
\hline & Volume(cc) & Dmean(Gy) & V5(\%) & V20(\%) & V30(\%) & V40(\%) \\
\hline Stomach & $256.71 \pm 140.73$ & & & & & \\
\hline Tangential & & $7.66 \pm 5.26$ & $21.15 \pm 16.98$ & $13.31 \pm 12.15$ & $10.27 \pm 9.70$ & $7.46 \pm 7.28$ \\
\hline IMRT & & $7.54 \pm 3.82$ & $49.62 \pm 22.65$ & $7.17 \pm 13.93$ & $1.31 \pm 2.09$ & $0.20 \pm 0.38$ \\
\hline p-value & & 0.89 & 0.01 & 0.04 & 0.02 & 0.02 \\
\hline Thyroid & $13.39 \pm 4.44$ & & & & & \\
\hline Tangential & & $16.34 \pm 7.89$ & $40.93 \pm 14.51$ & $30.59 \pm 16.92$ & $26.53 \pm 17.05$ & $22.37 \pm 16.58$ \\
\hline IMRT & & $15.34 \pm 3.71$ & $88.86 \pm 10.37$ & $23.92 \pm 13.49$ & $7.93 \pm 4.04$ & $2.63 \pm 2.32$ \\
\hline p-value & & 0.78 & 0.02 & 0.40 & 0.01 & 0.01 \\
\hline Right breast & $347.57 \pm 87.43$ & & & & & \\
\hline Tangential & & $0.48 \pm 0.43$ & $1.38 \pm 2.28$ & $0.23 \pm 0.40$ & $0.11 \pm 0.25$ & $0.05 \pm 0.13$ \\
\hline IMRT & & $6.16 \pm 1.49$ & $42.06 \pm 17.08$ & $1.03 \pm 2.31$ & $0.10 \pm 0.28$ & 0 \\
\hline p-value & & 0.01 & 0.01 & 0.25 & - & - \\
\hline Left lung & $923.57 \pm 281.58$ & & & & & \\
\hline Tangential & & $23.53 \pm 2.98$ & $59.88 \pm 5.40$ & $46.77 \pm 6.55$ & $43.54 \pm 7.05$ & $37.81 \pm 6.88$ \\
\hline IMRT & & $23.94 \pm 2.22$ & $93.71 \pm 4.48$ & $52.30 \pm 8.61$ & $31.53 \pm 3.63$ & $17.87 \pm 2.87$ \\
\hline p-value & & 0.21 & 0.01 & 0.01 & 0.01 & 0.01 \\
\hline Right lung & $1142.34 \pm 282.17$ & & & & & \\
\hline Tangential & & $0.52 \pm 0.14$ & $0.04 \pm 0.08$ & 0 & 0 & 0 \\
\hline IMRT & & $5.48 \pm 0.79$ & $34.49 \pm 9.77$ & $0.15 \pm 0.22$ & 0 & 0 \\
\hline$p$-value & & 0.01 & 0.01 & - & - & - \\
\hline
\end{tabular}

Dmean, mean dose; IMRT, Intensity Modulated Radiation Therapy. 
Jang et al. / Secondary cancer risk in breast cancer treated with radiotherapy

Table 3. Factors for estimating secondary cancer risk

\begin{tabular}{|c|c|c|c|c|c|}
\hline & OED(Gy) & mOED(Gy) & ERR & EAR & LAR \\
\hline \multicolumn{6}{|l|}{ Stomach } \\
\hline Tangential & $3.24 \pm 1.04$ & $1.94 \pm 0.44$ & $0.93 \pm 0.21$ & $9.49 \pm 2.16$ & $1.76 \pm 0.43$ \\
\hline IMRT & $4.75 \pm 1.12$ & $2.54 \pm 0.41$ & $1.22 \pm 0.19$ & $12.46 \pm 1.99$ & $2.31 \pm 0.42$ \\
\hline p-value & 0.01 & 0.01 & 0.01 & 0.01 & 0.01 \\
\hline \multicolumn{6}{|l|}{ Thyroid } \\
\hline Tangential & $3.04 \pm 0.78$ & $1.85 \pm 0.37$ & $1.95 \pm 0.39$ & - & $5.30 \pm 2.17$ \\
\hline IMRT & $6.85 \pm 0.74$ & $3.27 \pm 0.23$ & $3.43 \pm 0.24$ & - & $9.50 \pm 3.65$ \\
\hline p-value & 0.01 & 0.01 & 0.01 & - & 0.01 \\
\hline \multicolumn{6}{|l|}{ Right breast } \\
\hline Tangential & $1.12 \pm 0.21$ & $0.87 \pm 0.13$ & $0.44 \pm 0.06$ & $8.15 \pm 1.19$ & $0.35 \pm 0.08$ \\
\hline IMRT & $4.62 \pm 0.67$ & $2.51 \pm 0.25$ & $1.28 \pm 0.13$ & $23.56 \pm 2.38$ & $0.99 \pm 0.17$ \\
\hline p-value & 0.01 & 0.01 & 0.01 & 0.01 & 0.01 \\
\hline \multicolumn{6}{|l|}{ Left lung } \\
\hline Tangential & $4.16 \pm 0.30$ & $2.34 \pm 0.12$ & $3.27 \pm 0.17$ & $7.95 \pm 0.40$ & $1.68 \pm 0.15$ \\
\hline IMRT & $6.99 \pm 0.28$ & $3.32 \pm 0.09$ & $4.65 \pm 0.12$ & $11.28 \pm 0.30$ & $2.39 \pm 0.23$ \\
\hline p-value & 0.01 & 0.01 & 0.01 & 0.01 & 0.01 \\
\hline \multicolumn{6}{|l|}{ Right lung } \\
\hline Tangential & $1.04 \pm 0.06$ & $0.81 \pm 0.04$ & $1.14 \pm 0.05$ & $2.77 \pm 0.12$ & $0.58 \pm 0.05$ \\
\hline IMRT & $4.16 \pm 0.41$ & $2.42 \pm 0.16$ & $3.38 \pm 0.22$ & $8.21 \pm 0.54$ & $1.73 \pm 0.17$ \\
\hline p-value & 0.01 & 0.01 & 0.01 & 0.01 & 0.01 \\
\hline
\end{tabular}

OED, organ equivalent dose; ERR, excess relative risk; EAR, excess absolute risk; LAR, lifetime attributable risk; IMRT, Intensity Modulated

Radiation Therapy. Assuming an age at exposure of 30 years and an attained age of 60 years. LAR values are expressed per 100 persons.

Table 4. Monitor units and homogeneity indices.

\begin{tabular}{|c|c|c|c|c|c|c|}
\hline No & \multicolumn{2}{|c|}{$\begin{array}{c}\text { Monitor units } \\
\text { Tangential IMRT }\end{array}$} & \multicolumn{2}{c|}{$\begin{array}{c}\text { Homogeneity } \\
\text { indices }\end{array}$} & p-value \\
Tangential IMRT & \\
\hline Mean & 475.88 & 1369.75 & 0.01 & 0.29 & 0.14 & 0.01 \\
\hline 1 & 473 & 1404 & & 0.29 & 0.21 & \\
\hline 2 & 478 & 1073 & & 0.24 & 0.11 & \\
\hline 3 & 491 & 1585 & & 0.34 & 0.14 & \\
\hline 4 & 487 & 1454 & & 0.28 & 0.14 & \\
\hline 5 & 445 & 1075 & & 0.31 & 0.14 & \\
\hline 6 & 483 & 1401 & & 0.25 & 0.15 & \\
\hline 7 & 469 & 1324 & & 0.31 & 0.13 & \\
\hline 8 & 481 & 1642 & & 0.27 & 0.12 & \\
\hline
\end{tabular}

IMRT, intensity modulated radiation therapy.

\section{DISCUSSION}

Several studies have calculated the SC risks of RT in patients with BC (7-9), but few have performed analyses by stage in specific clinical situation. In the present study, we analyzed actual SC risks for specific organs to aid treatment decision making.

Thyroid cancer is known to be associated with BC (16), presumably because RT is widely used as a standard treatment for BC. Reportedly, BC survivors are at $10-50 \%$ higher risk of developing non-breast SC than members of the general population (17). In particular, if regional nodes are included in RT targets, the thyroid 926 may be exposed to substantial radiation doses. In the present study, we analyzed BC patients treated for regional nodes such as axillary and supraclavicular nodes. Calculated LAR values for thyroid cancer for 3D-CRT and IMRT were 5.3 (per 100 persons) and 9.5, respectively. The stomach is included in PTVs during RT of left BC, but there is little concern about toxicity, because RT-induced toxicities associated with stomach exposure are not as severe as dyspepsia or nausea. However, our calculations showed LAR of stomach SC risk was almost as great as that of thyroid and similar to that of the left lung. Many clinicians are less interested in radiation exposure of thyroid and stomach during RT for BC. However, according to our results, the SC of thyroid and stomach should not be overlooked during RT including regional nodes, and SC risk should be considered when deciding on RT technique.

IMRT is effective at reducing toxicities associated with high exposure doses $(4,5)$, but requires several times more MUs than tangential techniques. According to a study published by the Danish Breast Cancer Cooperative Group (DBCG), 9\% of SCs among women with early BC were attributable to radiation exposure (18), and other similar studies have also reported that $6-9 \%$ of SCs were caused by RT $(19,20)$. In the Int. J. Radiat. Res., Vol. 19 No. 4, October 2021 
present study, we assumed that SC develops at 60 years after exposure to radiation at 30 years of age, and we calculated LAR values of 9.67 (per 100 persons) and 16.92 for 3D-CRT and IMRT, respectively. To decrease MUs used for IMRT, Volumetric Modulated Arc Therapy (VMAT) may be an alternative. Although VMAT can reduce MU values as compared with IMRT, non-targeted organs are exposed to low doses (6), and extensive exposure to low doses has also been linked to SC. In one study, $58 \%$ of SCs occurred in areas exposed to $<6 \mathrm{~Gy}, 35 \%$ in areas exposed to 10-30 Gy, and 23\% occurred in areas exposed to $<1 \mathrm{~Gy}(20)$. Thus, in analyses of $\mathrm{SC}$, volumes exposed to low dose and MUs are important risk factors. In the present study, V5 of the IMRT technique was significantly greater for all organs than V5 of the tangential technique, and this disparity cannot be resolved using the VMAT technique. To address this topic, tangential IMRT has been attempted (22), but it is unlikely to be differentiated from conventional tangential RT. Therefore, we considered conventional tangential RT using the field in field technique was more practical from the clinical standpoint.

In practice, there is considerable skepticism about the application of the VMAT technique for BC RT. In one study, it was concluded, the VMAT technique presents a higher heart toxicity risk than the IMRT technique for RT of left BC (23). In $\mathrm{BC}$, the arc technique is likely to result in the exposure of a range of normal organs to low doses. In addition, set-up errors due to short treatment times in breasts with high respiratory movements present risks. Furthermore, during RT of large targets such as BC, respiratory-gated VMAT can cause multileaf collimator movement problems. For these reasons, VMAT technique was not included in the present study.

This study was conducted with the aim of providing information useful in clinical practice, and we selected controversial topic for RT techniques as study subjects. Conventional tangential techniques are commonly used for breast only treatment targets. However, in patients with RT targets that include the IMN, the IMRT technique can be applied to reduce heart and lung toxicities $(4,5)$. In patients with an IMN requiring treatment, there is sufficient justification to use the IMRT technique, but in patients requiring regional node treatment but not IMN treatment, IMRT has no significant benefit in terms of heart toxicity. IMRT tends to be applied indiscriminately without sufficient evidence of its merits and demerits in patients requiring regional node treatment without IMN treatment, and therefore, in the present study, we enrolled BC patients of this type.

We calculated mOEDs from organ OEDs and used them to evaluate SC risk. Since conventional RT is conducted using multiple fractions, BED was applied to correct fractional doses, and as a result, calculated mOEDs were about half the values of OEDs. The International Commission on Radiological Protection (ICRP) recommended a dose and dose rate effectiveness factor (DDREF) of two for extrapolation from high doses (24), and thus, our calculation results appear reasonable. Differences observed between patient OED values were attributed to body shapes and anatomical structures and suggest the need for individualized treatment. Furthermore, individual patient differences in terms of organ location and shape result in exposure differences, and thus, different SC risks, which indicates decisions that a particular RT technique is more suitable at a particular disease stage may be ill-founded. In the present study, HI values were better for the IMRT technique than the tangential technique, but it is questionable whether this observed improvement is clinically meaningful.

Consensus on the priority among disease control rate, SC potential, and toxicity is needed. Furthermore, in practice, the acceptability of an increased risk of SC is in BC patients with a low probability of severe toxicity and long life expectancy should be considered. SC is of greater importance for cancers with a low age at onset and a long life expectancy, such as are encountered in breast cancer. Therefore, when considering RT technique for BC, decisions should be made based on considerations of toxicity, age, and secondary cancer risk. We also suggest a program be devised that provides accurate information of the potential risk of SC in $\mathrm{BC}$ patients treated with RT. 
Certificate number of local institutional review board: 110757-201811-HR-03-02

\section{Conflicts of interest: Declared none.}

\section{REFERENCES}

1. Waks AG and Winer EP (2019) Breast cancer treatment: A review. JAMA, 321(3): 288-300.

2. Speers $C$ and Pierce $\amalg$ (2016) Postoperative radiotherapy after breast-conserving surgery for early-stage breast cancer: A review. JAMA Oncol, 2(8): 1075-1082.

3. Mo JC, Huang J, Gu WD, Gao M, Ning ZH, Mu JM, Li QL, Pei $\mathrm{HL}$ (2017) A dosimetric comparison of double-arc volumetric arc therapy, step-shoot intensity modulated radiotherapy and 3d-Crt for left-sided breast cancer radiotherapy after breast-conserving surgery. Technol Health Care, 25 (5): 851-858.

4. Finazzi T, Nguyen VT, Zimmermann F, Papachristofilou A (2019) Impact of Patient and Treatment Characteristics on Heart and Lung Dose in Adjuvant Radiotherapy for LeftSided Breast Cancer. Radiat Oncol, 14(1): 153.

5. Dumane VA, Bakst R, Green S (2018) Dose to organs in the supraclavicular region when covering the internal mammary nodes (Imns) in breast cancer patients: A comparison of volumetric modulated arc therapy (Vmat) versus 3d and vmat. PLoS One, 13(10): e0205770.

6. Karpf D, Sakka M, Metzger M, Grabenbauer GG (2019) Left breast irradiation with tangential intensity modulated radiotherapy (T-Imrt) versus tangential volumetric modulated arc therapy (T-Vmat): Trade-offs between secondary cancer induction risk and optimal target coverage. Radiat Oncol, 14(1): 156.

7. Momeni Z, Tavakoli MB, Atarod M (2018) Estimation of the thyroid secondary cancer risk on the patient of standard breast external beam radiotherapy. J Med Signals Sens, 8(4): 238-243.

8. Kim DW, Chung WK, Shin D, Hong S, Park SH, Park SY, Chung K, Lim YK, Shin D, Lee SB, Lee HH, Yoon M (2013) Risk of second cancer from scattered radiation of intensity -modulated radiotherapies with lung cancer. Radiat Oncol, 8: 47.

9. Lee B, Lee S, Sung J, Yoon M (2014) Radiotherapy-induced secondary cancer risk for breast cancer: $3 d$ conformal therapy versus imrt versus vmat. J Radiol Prot, 34(2): 325331.

10. Vlachopoulou V, Malatara G, Delis H, Kardamakis D, Panayiotakis $G$ (2013) Estimation of the risk of secondary cancer in the thyroid gland and the breast outside the treated volume in patients undergoing brain, mediastinum and breast radiotherapy. Radiat Prot Dosimetry, 154(1): 121-126.
11. Schneider U, Zwahlen D, Ross D, Kaser-Hotz B (2005) Estimation of radiation-induced cancer from threedimensional dose distributions: Concept of organ equivalent dose. Int J Radiat Oncol Biol Phys, 61(5): 1510-1515.

12. Jones B, Dale RG, Deehan C, Hopkins KI, Morgan DA (2001) The role of biologically effective dose (BED) in clinical oncology. Clin Oncol, 13(2): 71-81.

13. Gregoire V and Mackie TR (2011) State of the art on dose prescription, reporting and recording in intensitymodulated radiation therapy (Icru Report No. 83). Cancer Radiother, 15(6-7): 555-559.

14. Donovan EM, James $H$, Bonora M, Yarnold JR, Evans PM (2012) Second cancer incidence risk estimates using beir Vii models for standard and complex external beam radiotherapy for early breast cancer. Med Phys, 39(10): 58145824.

15. Preston DL, Mattsson A, Holmberg E, Shore R, Hildreth NG, Boice JD, Jr. (2002) Radiation effects on breast cancer risk: A pooled analysis of eight cohorts. Radiat Res, 158(2): 220235.

16. Nielsen SM, White MG, Hong $S$, Aschebrook-Kilfoy B, Kaplan EL, Angelos P, Kulkarni SA, Olopade OI, Grogan RH (2016) The breast-thyroid cancer link: A systematic review and meta-analysis. Cancer Epidemiol Biomarkers Prev, 25 (2): 231-238.

17. Rubino C, de Vathaire F, Diallo I, Shamsaldin A, Le MG (2000) Increased risk of second cancers following breast cancer: Role of the initial treatment. Breast Cancer Res Treat, 61(3): 183-195.

18. Grantzau T, Mellemkjaer L, Overgaard J (2013) Second primary cancers after adjuvant radiotherapy in early breast cancer patients: A national population based study under the danish breast cancer cooperative group (Dbcg). Radiother Oncol, 106(1): 42-49.

19. Berrington de Gonzalez A, Curtis RE, Gilbert E, Berg CD, Smith SA, Stovall M, Ron E (2010) Second solid cancers after radiotherapy for breast cancer in seer cancer registries. Br J Cancer, 102(1): 220-226.

20. Maddams J, Parkin DM, Darby SC (2011) The cancer burden in the United Kingdom in 2007 due to radiotherapy. Int J Cancer, 129(12): 2885-2893.

21. Dorr W and Herrmann T (2002) Cancer Induction by radiotherapy: Dose dependence and spatial relationship to irradiated volume. J Radiol Prot, 22(3A): A117-121.

22. Abo-Madyan $Y$, Aziz MH, Aly MM, Schneider F, Sperk E, Clausen S, Giordano FA, Herskind C, Steil V, Wenz F, Glatting G (2014) Second cancer risk after 3d-Crt, imrt and vmat for breast cancer. Radiother Oncol, 110(3): 471-476.

23. Sakka M, Kunzelmann L, Metzger M, Grabenbauer GG (2017) Cardiac dose-sparing effects of deep-inspiration breath-hold in left breast irradiation : Is imrt more beneficial than vmat? Strahlenther Onkol, 193(10): 800-811.

24. ICRP (2007) The 2007 Recommendations of the International Commission on Radiological Protection. Icrp Publication 103. Ann ICRP, 37(2-4): 1-332. 